\title{
Corticobasal ganglionic degeneration and/or frontotemporal dementia? A report of two overlap cases and review of literature
}

\author{
P S Mathuranath, John H Xuereb, Thomas Bak, John R Hodges
}

\begin{abstract}
Objective-According to the existing viewpoint, Corticobasal degeneration (CBD) is thought of as a predominantly extrapyramidal motor disorder that is distinct and unrelated to frontotemporal dementia (FTD), the most common form of non-Alzheimer dementias. A lack of understanding of the aetiopathogenesis, and poor correlation between the pathology and the clinical syndromes, has resulted in a disparity in the classification of cases of non-Alzheimer dementias. This report intends to highlight the overlap between FTD and CBD in the light of the evolution of these terms, and to discuss the implications of these findings on the nosology of CBD and the classification of non-Alzheimer dementias.

Methods and results-Two cases who presented with cognitive dysfunction, which, on comprehensive neuropsychological testing warranted an antemortem diagnosis of FTD are reported. A detailed necropsy study of their brains, however, favoured a pathological diagnosis of CBD. The literature on the overlap between CBD and FTD is also reviewed.

Conclusions-Firstly, evidence is emerging to suggest that the clear distinction drawn between FTD and CBD by the existing viewpoint, needs revision. Secondly, until such time that a comprehensive classification of non-Alzheimer dementias is evolved, it may be better to distinguish between the clinical and pathological levels of description and to classify cases, in vivo, on the basis of the clinical phenotype.

(F Neurol Neurosurg Psychiatry 2000;68:304-312)
\end{abstract}

Keywords: corticobasal degeneration; frontotemporal dementia; frontotemporal lobar degeneration; nonAlzheimer dementias

Corticobasal degeneration (CBD) is currently considered by many authors to be a distinct clinicopathological entity. It is distinguished from frontotemporal dementia (FTD) and other neurodegenerative diseases. According to this viewpoint the major features of $\mathrm{CBD}$ are extrapyramidal (akinetic-rigidity and dystonia) with apraxia, alien limb phenomenon, and other features (myoclonus, tremor, and rarely oculomotor and bulbar abnormalities). Frank dementia or language dysfunctions are said to be rare and if present are mild, and typically occur late in the course of the disease. ${ }^{1}$ Pathologically CBD is characterised by asymmetric cortical atrophy predominantly in the perirolandic area. Histology is the gold standard for the diagnosis of CBD. It is distinguished by achromatic ballooned neurons that stain positive with tau-immunohistochemical stains. tau-positive inclusions are also found in glial cells in the atrophic areas of the cortex. Neuronal loss and gliosis with basophilic inclusions are prominent in the substantia nigra and pallidum. The other subcortical structures involved include the caudate, putamen, subthalamus, and the dentate nucleus. ${ }^{2}$ It is increasingly apparent, however, that there is not complete concordance between the clinical syndrome and pathological findings. ${ }^{3-16}$ We report on two patients with histologically confirmed CBD with atypical clinical presentation of frontotemporal dementia (FTD), one of whom had prominent aphasia. Both patients underwent comprehensive neuropsychological evaluation of the type applied by our group to other neurodegenerative disorders ${ }^{17-21}$ but hitherto not used in CBD. We discuss the implications of the present findings for the nosology of CBD and the overlap between non-Alzheimer forms of dementias.

Case 1

A 67 year old woman was first seen in November 1991 by her general practitioner with a short history of reduced conversation and agitated behaviour. She was started on a low dose of dothiepin, which improved her sleep but not her agitation. Hence, it was stopped after 3 months. In March 1992 she was referred to the psychiatric services because she had become very hyperactive at home with poor concentration, was overeating, and had a compulsive desire to micturate often. Examination then showed disinhibited behaviour and markedly impaired concentration. She scored 20/30 on the mini mental state examination (MMSE) with difficulties in orientation, immediate memory, and simple drawings. There were no perceptual abnormalities, psychotic features, or mood changes. In August 1992, she was seen in the memory clinic at the Addenbrooke's Hospital for the first time. By now there was a marked change in her personality and behaviour, apathy, sparse spontaneous conversation, inability to plan and organise, impulsivity, and occasional aggression towards family members. She was obsessed with watching television, totally neglectful of her housework, and would occasionally lose her way in the house. 
Table 1 Results of neuropsychological tests

\begin{tabular}{|c|c|c|c|}
\hline Tests (maximum score) & Case 1 & Case 2 & Controls* \\
\hline Year of testing & Oct 1992 & Feb 1993 & \\
\hline No of years of education & 9 & 9 & $10.8(2.2)$ \\
\hline Handedness & Right & Right & \\
\hline NART (Nelson, 1982; errors, 50) & 33 & 33 & $10.9(6.2)$ \\
\hline MMSE (30) & 20 & 10 & $29.2(1.0)$ \\
\hline \multicolumn{4}{|l|}{ Memory } \\
\hline \multicolumn{4}{|l|}{ Logical memory (WMS) (mean score) } \\
\hline Immediate recall (23.5) & 2 & NA & $23.2(7.8)$ \\
\hline Delayed recall (23.5) & 0.5 & NA & $17.0(6.8)$ \\
\hline \multicolumn{4}{|l|}{ Warrington recognition memory test } \\
\hline Words (50) & 25 & NA & $47.3(2.8)$ \\
\hline Faces (50) & 34 & 24 & \\
\hline \multicolumn{4}{|l|}{ Rey figure } \\
\hline Copy (36) & 7 & 30 & $44.6(20.7)$ \\
\hline$\%$ Recall & $7(100 \%)$ & 0 & $15.2(7.4)$ \\
\hline Digit span (WMS total score) & 11 & 4 & \\
\hline \multicolumn{4}{|l|}{ Visuoperceptual tests } \\
\hline Benton line orientation (30) & 5 & Failed practice & $27.4(4.0)$ \\
\hline Object matching unusual views (40) & 33 & NA & $37.3(3.1)$ \\
\hline Object decision (64) & 32 & 35 & \\
\hline \multicolumn{4}{|l|}{ Language } \\
\hline Token test (36) & 23.5 & 9 & $35.7(0.5)$ \\
\hline \multicolumn{4}{|l|}{ TROG } \\
\hline Blocks passed (20) & 6 & 2 & \\
\hline Total correct $(80)$ & 54 & 33 & $78.8(1.8)$ \\
\hline \multicolumn{4}{|l|}{ Reading-the surface list } \\
\hline Regular words (126) & $118(93.6 \%)$ & NA & $125.2(2.7)$ \\
\hline Exception words (126) & $110(87.3 \%)$ & NA & $123.6(3.1)$ \\
\hline Reading non-words (40) & 27 & 21 & $39.3(0.9)$ \\
\hline Writing (36) & 25 & 25 & $35.3(0.9)$ \\
\hline \multicolumn{4}{|l|}{ Semantic battery } \\
\hline Category fluency (correct responses) & $28 \dagger$ & $5 \dagger$ & $113.7(20.9)$ \\
\hline Letter fluency (correct responses) & 14 & 1 & $44.6(10.2)$ \\
\hline Naming (48) & 25 & 31 & $43.6(2.3)$ \\
\hline Naming to description (24) & 9 & 4 & $22.5(1.4)$ \\
\hline Word-picture matching (48) & 37 & 38 & $47.4(1.1)$ \\
\hline \multicolumn{4}{|l|}{ Category (Picture) Sorting } \\
\hline Level $1(48)$ & 37 & NA & $48.0(0.2)$ \\
\hline Level 2 (48) & 40 & NA & $46.9(0.9)$ \\
\hline Level $3(72)$ & 5 & NA & $68.8(2.2)$ \\
\hline \multicolumn{4}{|l|}{ Semantic features questions } \\
\hline 24 Standard items (192) & $139(72.3 \%)$ & NA & $178.0(5.1)$ \\
\hline Pyramids and palm trees (52) & $30(57.6 \%)$ & $18(34.6 \%)$ & $51.2(1.4)$ \\
\hline Raven coloured matrices (36) & $\mathrm{NA}$ & 17 & \\
\hline
\end{tabular}

${ }^{\star} \mathrm{n}=24$; figures in parentheses are $\mathrm{SD}$; †Predominant error was perserveration; WMS=Wechsler memory scale; TROG=test for the reception of grammar.

$\mathrm{NA}=$ not done.

She remained, cheerful and at times was even euphoric in her mood. The only significant medical history was cataract surgery in 1990. Her mother and father had died at 84 and 76 years of age, respectively. She had three siblings who were all healthy. There was no family history of dementia. Positive findings on general examination included a labile blood pressure fluctuating between 140/70 and 160/80 $\mathrm{mm}$ of

Table 2 Summary of pathological findings in the two cases

\begin{tabular}{|c|c|c|}
\hline & Case 1 & Case 2 \\
\hline \multicolumn{3}{|l|}{ Macroscopic } \\
\hline Posterior frontal lobe (BA 6,8) atrophy & +++ & +++ \\
\hline Frontal pole atrophy & + & + \\
\hline Precentral gyrus atrophy & + & \pm \\
\hline Anterior cingulate gyrus atrophy & +++ & ++ \\
\hline Temporal pole atrophy & ++ & ++ \\
\hline Parahippocampal gyrus atrophy & ++ & + \\
\hline Hippocampal formation atrophy & \pm & \pm \\
\hline Parietal lobe atrophy & + & + \\
\hline Occipital lobe atrophy & + & - \\
\hline Substantia nigra depigmentation and atrophy & +++ & +++ \\
\hline Caudate atrophy & ++ & + \\
\hline Pallidal atrophy & + & + \\
\hline Thalamic atrophy & - & - \\
\hline Subthalamic nucleus atrophy & - & - \\
\hline \multicolumn{3}{|l|}{ Microscopic } \\
\hline Ballooned achromatic neurons & +++ & +++ \\
\hline Tau+ve cytoplasmic neuronal inclusions $\star$ & ++ & ++ \\
\hline Glial cytoplasmic inclusions & ++ & ++ \\
\hline Basophilic neuronal cytoplasmic inclusions $\dagger$ & + & +++ \\
\hline Pale body neuronal cytoplasmic inclusionst & ++ & ++ \\
\hline
\end{tabular}

*In cortical layers IV/V; †in basal ganglia.

+++ Severe; ++ moderate; + mild; \pm doubtful; - absent.
Hg. Neurological examination showed a left sided dysgraphaesthesia, and right-left disorientation. No rigidity, postural instability, or gait abnormalities were present and she was able to perform clinical tests of praxis (transitive and intransitive gestures to commands) examination. Alien limb phenomenon was absent.

Her performance was impaired on all the neuropsychological tests administered, with marked deficits in episodic memory, language production and comprehension, and visuospatial abilities (table 1). There was evidence for frontal lobe type difficulties on a range of tests with poor understanding of the task demands and difficulties complying with relatively simple instructions. Routine haemogram and biochemical tests on blood were normal. A head CT in 1992 was reportedly normal (the films have subsequently been lost). A ${ }^{99} \mathrm{Tc}-\mathrm{HMPAO}$ SPECT in 1992 showed bilateral frontal hypoperfusion. A clinical diagnosis of advanced FTD was made on the basis of the history of prominent early change in personality and behaviour, plus the SPECT evidence of frontal dysfunction. Over the next 2.5 years her illness progressed. She developed marked hyperphagia and became extremely disinhibited and aggressive. She also had repeated falls and in December 1995 she died.

At necropsy, the brain weighed $900 \mathrm{~g}$ and showed symmetric frontotemporal gyral atrophy. The gross and microscopic pathological findings are summarised in table 2. Curiously, the most severe atrophy affected the posterior parts of the transverse (superior, middle, and inferior) frontal gyri (Brodmann area (BA) 6 and 8 ). The frontal pole (BA 9 and 10) and precentral gyrus (BA 4) were better preserved (fig 1). There was no evidence of a severe "knife edge" pattern of atrophy that is characteristic of Pick's disease. The temporal pole, anterior part of the parahippocampal gyrus, and parietal and occipital lobes also showed moderate atrophy, but the hippocampal formation was relatively spared. The white matter of the frontal lobe was gliotic and reduced in thickness, and the lateral ventricle was proportionately enlarged. The basal ganglia, particularly the caudate nucleus and the globus pallidus, were moderately atrophied. The substantia nigra was markedly pale. Microscopically, nerve cell loss paralleled the distribution of atrophy, and areas of severe neuronal loss showed spongiosis of the neuropil and reactive astrocytic gliosis. Numerous "ballooned achromatic neurons", which were tau and $\alpha \mathrm{B}$ crystallin positive on immunohistochemical preparations, were present in layers V/VI of affected cerebral areas, and were particularly numerous in the anterior cingulate cortex (fig 2). In addition, numerous tau positive, ubiquitin negative, small, curved, fibrillary, cytoplasmic inclusions were present in small neurons of the superficial layers of neocortex (layer II) (fig 3). Similar, but smaller and more slender cytoplasmic inclusions were present in glial cells within subcortical white matter (fig 4). Neuritic plaques, neurofibrillary tangles of Alzheimer-type and distribution, Pick bodies, 


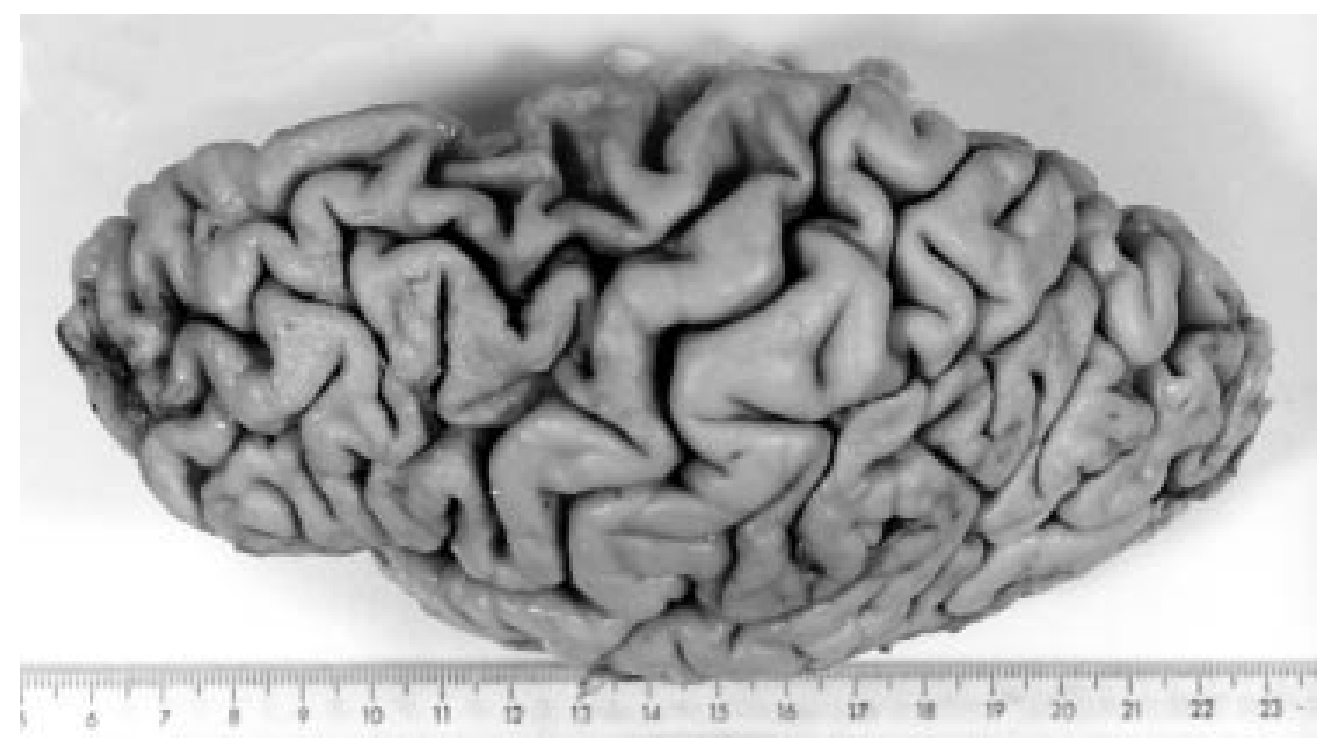

Figure 1 Superlateral view of the left cerebral hemisphere from case 1, showing the focal atrophy of cerebral gyri in BA 6.

and ubiquitin positive inclusions were absent from both hippocampus and neocortex. There was no evidence of Lewy body formation or basophilic nuclear inclusions in the pigmented brainstem nuclei, but a pale body cytoplasmic inclusion was present in a few of the remaining neurons of the substantia nigra (fig 5). The motor nuclei of the brainstem remained unaffected. A pathological diagnosis of corticobasal degeneration was made.

\section{Case 2}

A 60 year old woman presented to the memory clinic at Addenbrooke's Hospital in February 1993 with a history of progressive word finding difficulty over 2 years. Her day to day memory was reported to be reasonable by her husband. Until about a year previously she could still follow conversations, cook, and with some help, do shopping lists. Initially, she was quite insightful, becoming embarrassed about going

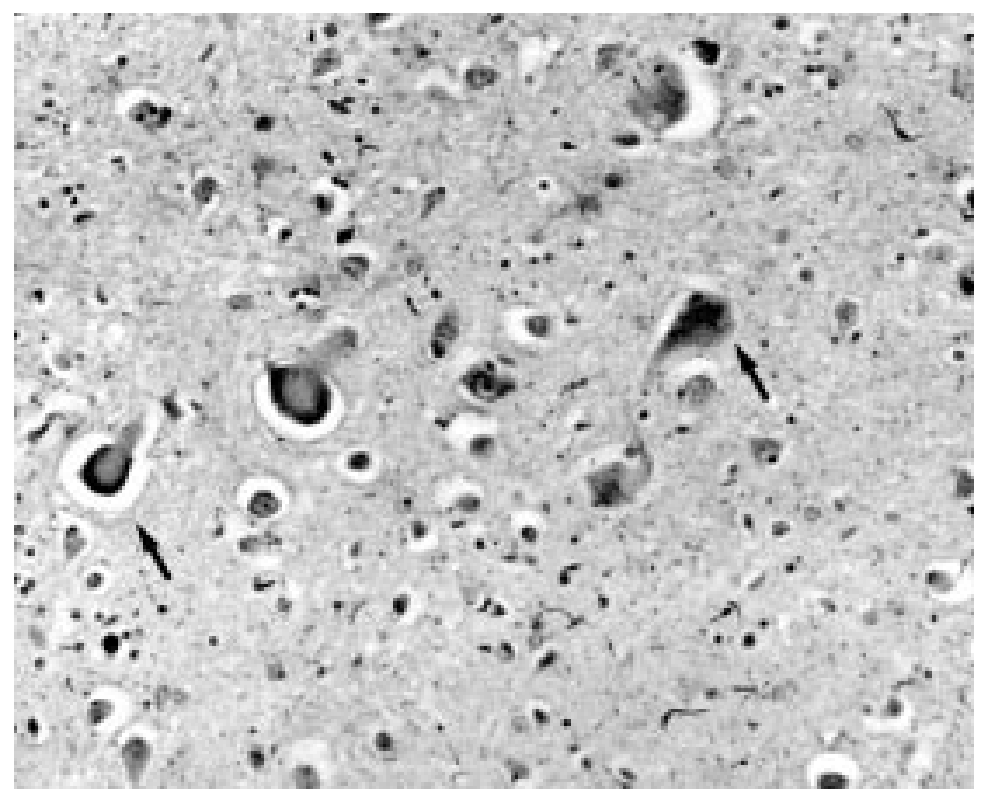

Figure 2 Photomicrograph of anterior cingulate cortex showing numerous ballooned achromatic neurons (arrows) in layers V/VI (tau-immunohistochemistry originally $\times 200)$. to the Artist's Guild and Women's Institute. Of late, her insight had declined and she had become restless and impulsive (for example, although she could prepare vegetables she would not wait for them to cook). She had developed marked verbal perseveration and echolalia. She had been taking $10 \mathrm{mg}$ temazepam at night for a considerable period of time. Otherwise her medical history was unremarkable. Her father had died at 70 and her mother was said to have had a dementing disorder and died at 82 years of age. She was the only child of her parents. Her two children were alive and well.

On examination, she had a marked fluent dysphasia with anomia, euphoria, perseveration, echolalia, and echopraxia. Comprehension was profoundly impaired and on the MMSE she scored 10/30. Salient findings on detailed neuropsychological testing (table 1) were impaired recognition memory test for faces, word fluency, naming, naming to description, reading, writing, and comprehension. Her ability to copy the Rey figure was, however, only mildly impaired. Neurological and general physical examination were unremarkable. Routine blood haemogram and biochemical tests were within normal limits and syphilis serology was negative. A 16 channel surface EEG awake record showed bilateral, medium amplitude theta activity in the frontotemporal regions (left more than right) intermixed with a posterior central background $\alpha$ activity of $9 \mathrm{~Hz}$. T1 weighted coronal and T2 weighted axial MR images of the brain showed mild generalised atrophy with more marked frontal atrophy, left more than right, minor left temporal pole atrophy, and normal hippocampi. T2 Weighted images showed some periventricular lucencies. A ${ }^{99} \mathrm{Tc}-\mathrm{HMPAO}$ SPECT showed bilateral frontotemporal hypoperfusion. In view of the disproportionately severe progressive fluent aphasia with moderate frontal dysfunction, FTD with mixed frontal and temporal lobe involvement was considered the most likely diagnosis. 


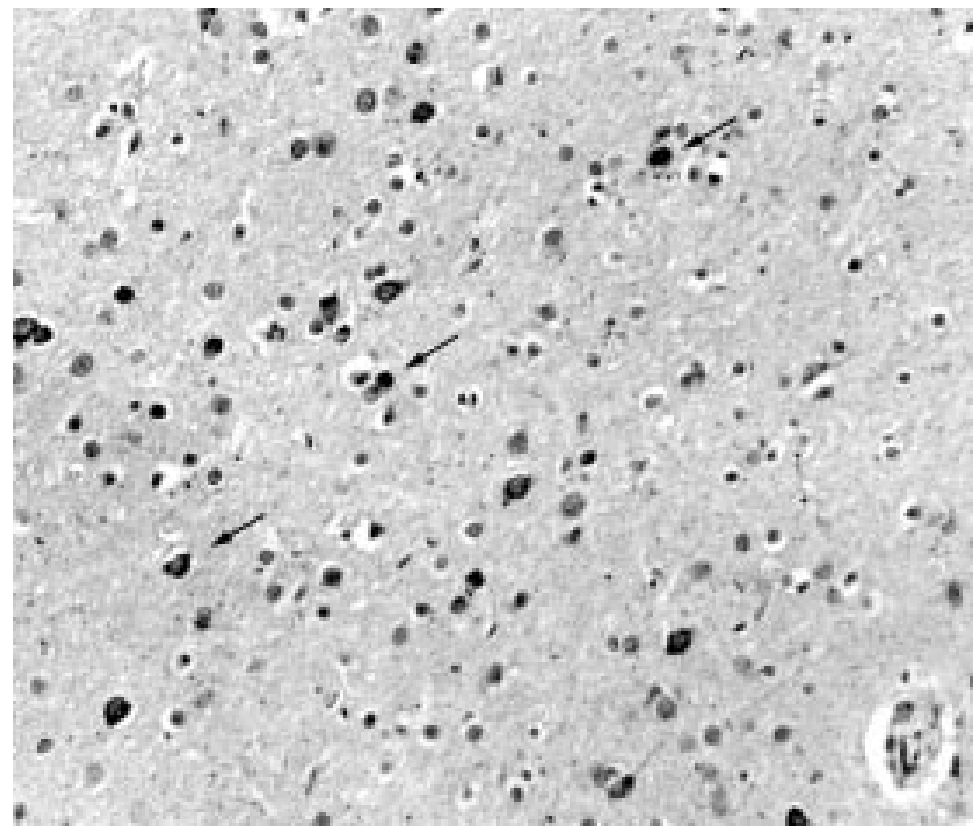

Figure 3 Photomicrograph of the cerebral cortex from BA6 showing numerous small, curved, fibrillary, cytoplasmic inclusions in small neurons (arrows) of the superficial layers of neocortex (layer II) (tau immunohistochemistry originally $\times 200$ ).

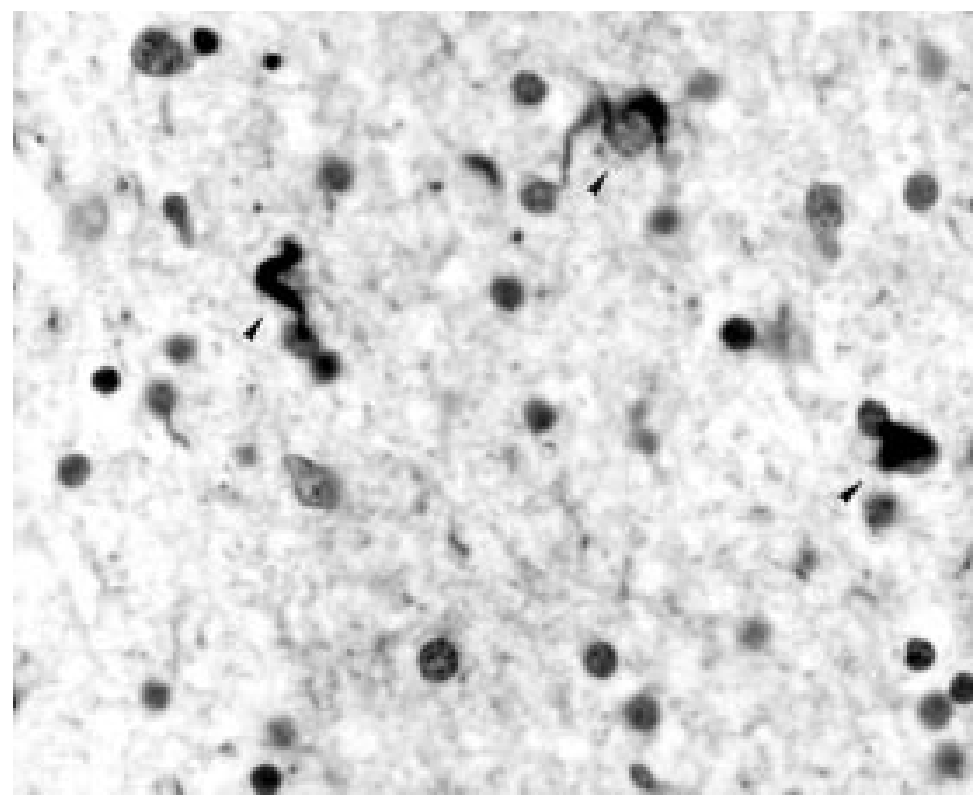

Figure 4 Photomicrograph of temporal neocortex showing cytoplasmic inclusions in glial cells (arrow heads) within subcortical white matter (tau immunohistochemistry originally $\times 800)$.

Over the next 3 years she showed progressive decline in her language and cognitive functions. She developed mild features of KluverBlucy syndrome in the form of oral tendencies and hyperphagia. She also developed frank emotional labiality. Of note is the fact that she failed to show any obvious involuntary movements, extrapyramidal signs, alien limb phenomemon, or features of apraxia until she died in November 1996.

The neuropathological changes (summarised in table 2) were similar to those recorded in the previous case. At necropsy the brain weighed $1200 \mathrm{~g}$. A frontotemporal pattern of atrophy was present, most severely involving the frontal lobe anterior to the precentral gyrus. As in case 1, the cerebral hemispheres were symmetrically involved, and the degree of atrophy did not approximate the severe "knife edge" atrophy seen in Pick's disease. The central white matter of the frontal lobe and the anterior part of the corpus callosum were reduced in thickness and gliotic, and the anterior horn of the lateral ventricle was enlarged as a result. The caudate and lentiform nuclei showed atrophy, but the thalamus and subthalamic nuclei were normal. The substantia nigra and locus coeruleus in the brainstem were pale. Microscopically, sections of cerebral neocortex from frontal, temporal, and parietal regions showed numerous tau and $\alpha \mathrm{B}$-crystallin positive "ballooned achromatic neurons" in deeper layers (IV and V). In layer II, curved or skeinlike, tau positive inclusions were present in small neurons. In subcortical white matter, numerous glial cells contained tau positive inclusions. Frontal cortex (BA 44/45, 6 and 10) and cingulate gyrus were most severely affected, with vacuolation of the superficial layer and subcortical gliosis, and the temporal cortex (BA 21 and 22) was moderately affected. Marked nerve cell loss was seen in the substantia nigra, without Lewy body formation in residual neurons. As in case 1 , an occasional neuron contained a pale inclusion surrounded by neuromelanin granules. In addition, two remaining neurons contained a slightly basophilic, faintly fibrillar cytoplasmic inclusion. Neurofibrillary inclusions of Alzheimer-type were present in pyramidal neurons of the pre- $\alpha$ layer of the entorhinal cortex and in a few neurons of the CA1 sector of the hippocampal pyramidal layer. Also in the superficial entorhinal cortex, an occasional nerve cell body contained a spherical circumscribed homogeneous tau positive inclusion, characteristic of Pick bodies. Neither these inclusions nor tau negative ubiquitin positive inclusions of the type seen in motor neuron disease were present in the dentate fascia. Neuritic plaques were absent. The brainstem motor nuclei were unremarkable. A neuropathological diagnosis of CBD was made. The mild degree of Alzheimer-type pathology (Braak neurofibrillary stage II) is not significant.

\section{Discussion}

CLINICAL DIAGNOSIS OF FTD

We have described here two patients with clinical features suggestive of FTD. Patient 1 started with predominant behavioural changes and soon evolved into frank dementia. The initial features point to frontal lobe dysfunction that was confirmed by the results of SPECT although the visuospatial deficits were atypical for FTD. Subsequent symptoms suggest medial temporal as well as parietal lobe involvement, as substantiated by the results of neuropsychological tests. Thus clinical and investigative findings were in keeping with a diagnosis of FTD. ${ }^{22}{ }^{23}$ Patient 2, by contrast, presented with a progressive aphasic syndrome which only later evolved to involve other cognitive domains. The findings of severe aphasia, with initial preservation of activities of daily living, might suggest a clinical diagnosis of pri- 


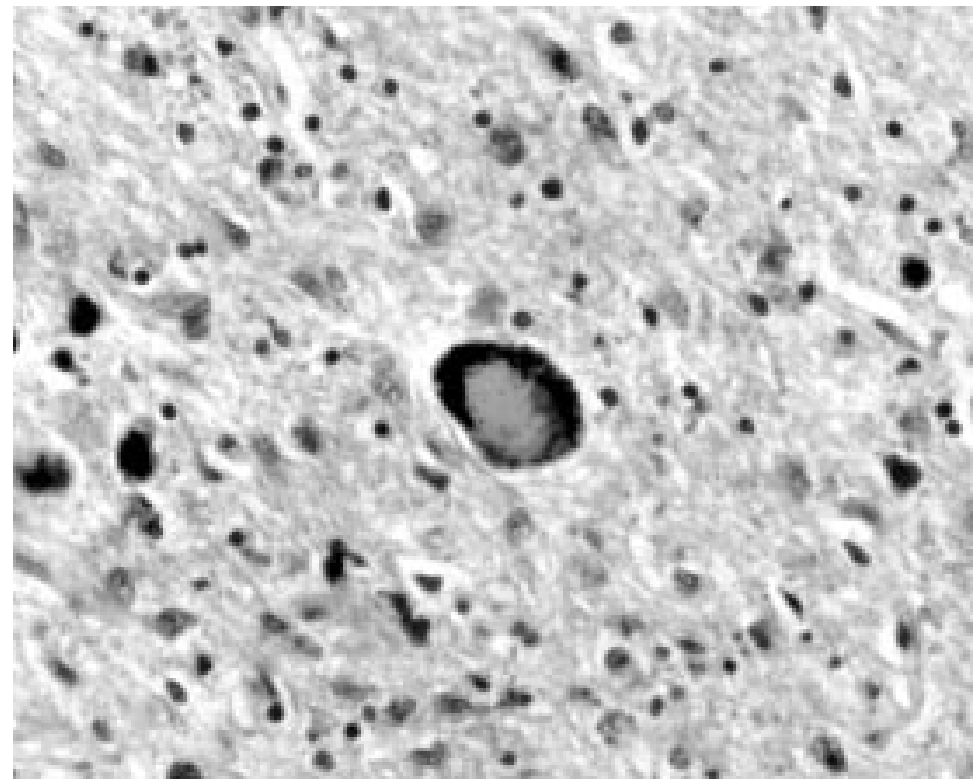

Figure 5 Photomicrograph of substantia nigra showing a pale inclusion in one of the few of the remaining neurons (haematoxylin and eosin originally $\times 200$ ).

mary progressive aphasia. ${ }^{24}$ By the time of assessment, however, she was exhibiting clear features of frontal dysfunction (for example, impulsivity and perseveration) and showed cognitive deficits on formal neuropsychological testing beyond the domain of language. She did not, therefore, fulfil strict diagnostic criteria for primary progressive aphasia, which require normal activities of daily living and performance on non-linguistic tasks. ${ }^{25}$ By contrast, the combination of severe aphasia with behavioural deficits fits better within the clinical range of FTD. ${ }^{26}$ It is worth noting that neither of the patients showed signs of ideomotor apraxia, alien-limb phenomenon, extrapyramidal signs, myoclonus, or delayed postural instability, the typical features of CBD. There was, therefore, little reason to suspect clinically a diagnosis of CBD.

PATHOLOGICAL DIAGNOSIS OF CBD

In the two cases that are the subject of this report, the neuropathological diagnosis of CBD, was based on the following features: $(a)$ relatively circumscribed cortical degeneration, characterised microscopically by numerous small, superficial cortical, tau-positive ubiquitin negative intraneuronal inclusions, but with neither neuritic plaque formation nor neurofibrillary inclusions in pyramidal neurons of layers IV/V, (b) numerous ballooned achromatic neurons, but virtually absent Pick bodies, (c) glial cell cytoplasmic inclusions, and $(d)$ severe substantia nigra cell loss with pale inclusions and occasional basophilic inclusions, but no Lewy bodies or neurofibrillary tangles.

In both cases the cerebral cortical atrophy was more prominent than the atrophy of the corpus striatum, by contrast with the pattern usually seen in cases of multisystem atrophy (MSA) (striatonigral degeneration). Oligodendroglial cytoplasmic inclusions (GCIs) were considered diagnostic of MSA when first described, ${ }^{27}$ but have since been described in several other neurodegenerative diseases, including CBD. In CBD, as in our cases, the GCIs are most often found in the white matter that underlies the affected cerebral cortex. ${ }^{28} 29$ The minimal involvement of the entorhinal cortex and hippocampus, the absence of neuritic plaques, and the absence of typical neurofibrillary inclusions in neocortical pyramidal neurons in layers IV/V, militate against a diagnosis of Alzheimer's disease. Progressive supranuclear palsy (PSP) enters the differential diagnosis because tau positive fibrillary intraneuronal inclusions and ballooned achromatic neurons are seen in this disease. However, the NINDS neuropathological criteria for typical PSP require the presence of numerous neurofibrillary inclusions in at least three of the following areas: pallidum, subthalamic nucleus, substantia nigra, and pons. ${ }^{30}$ Even "atypical PSP" requires some neurofibrillary tangle formation in the basal ganglia and brainstem. None of these areas contained fibrillary inclusions in the two cases that are the subject of this report. Frontal lobe degeneration may be seen in motor neuron disease, when it may manifest as late stage bulbar involvement. This condition can be identified by ubiquitin positive, tau negative, cytoplasmic inclusions in the small neurons of the dentate gyrus, by the presence of neuronal loss from motor nuclei, from the hypoglossal nucleus and from the motor cortex, and by the absence of ballooned neurons and of neuronal and glial tau positive inclusions. Clearly, this diagnosis can be excluded in the two cases of this report, as none of these features were present.

Pick's disease is also an inappropriate diagnosis because these cases lack severe "knife edge" cerebral gyral atrophy and severe cortical neuronal loss in the affected cortex, features which characterise this disease. ${ }^{31}$ In classic Pick's disease, numerous cortical Pick bodies and variable numbers of achromatic ballooned neurons are present. Cases do occur in which focal cortical atrophy and severe nerve cell loss are present, but which lack either Pick bodies or Pick cells. Such cases have been called nonPick's lobar atrophy by some, ${ }^{32}$ whereas others accept them as Pick's disease, based on the pattern of atrophy and the absence of histological features of other dementing conditions. Many neuropathologists will give such cases the label of "atypical” Pick's disease. Hulette and Crain ${ }^{33}$ noted that, unlike neurofibrillary tangles, Pick bodies do not remain in the extracellular space after death of the nerve cell, which may explain the absence of Pick bodies in otherwise typical cases. In the two cases that are the subject of this report, nerve cell loss was not severe, so that the virtual absence of Pick bodies may be due to a pathogenic mechanism that is different from that of Pick's disease. Indeed, in pathological terms, these cases are more closely allied to CBD than to Pick's disease, as they differ from classic cases of CBD only in terms of the cortical area that is affected. Like classic CBD, the principal areas of CNS involved in the degenerative process are the cerebral cortex, basal ganglia, and sub- 
stantia nigra; also, the specific combination and pattern of histological lesions are identical.

RELATION OF CBD AND FTD

To appreciate the relation between $\mathrm{CBD}$ and FTD it is necessary to review evolving terminologies and the underlying concepts in the field. A lack of detailed understanding of the aetiopathogenesis and poor correlation between the pathology and the clinical syndromes in non-Alzheimer dementias, has led to a plethora of terminologies and the resulting confusions in its nosology. Whereas some terms apply to a clinical syndrome without implications of a specific histology (for example, progressive non-fluent aphasia, semantic dementia), others imply a distinct pathology (for example, Pick's disease) and still others combine features of both (for example, FTD, CBD). Pick was the first to draw attention to the occurrence of a predominantly behavioural focal cognitive presentation of neurodegenerative disease when he provided elegant descriptions of patients with bilateral frontal and temporal atrophy (translations of the original papers of Arnold Pick by Berrios and Girling $^{34}$ ). A few years later Alzheimer discovered intraneuronal inclusions and ballooned neurons, which were subsequently referred to as Pick bodies and Pick cells, respectively. ${ }^{35}$ After this, before the second world war, the term "Pick's disease" was used for patients whose clinical syndrome of frontal dysfunction had an underlying pathology of frontal atrophy with Pick cells and Pick bodies, although, some used it for any patient with the syndrome of frontal dysfunction. Sadly, the temporal pattern of involvement was completely neglected. ${ }^{36}$ In the next phase, the emphasis shifted to pathology. Patients with the same clinical syndrome of frontal lobe dysfunction but without the specific histological changes (of Pick cells and Pick bodies) were referred to by various names. These include dementia of frontal type ${ }^{2637}$; frontal lobe degeneration of the non-Alzheimer's type ${ }^{38}$; frontal lobe degeneration $^{39}$; and dementia lacking distinctive features. ${ }^{40}$ The factors that remained common to all these patients was a clinical syndrome characterised by profound behavioural disorder and change in personality and social conduct and a topographical distribution of the degeneration involving bilateral frontal and temporal lobes.

In parallel with the above development was the rekindling of interest in a different clinical syndrome, also resulting from focal cortical atrophy. Unlike the predominantly behavioural frontal lobe syndrome discussed above, the syndrome of primary progressive aphasia ${ }^{24}$ was characterised by an initial decline in language function with relatively late and less severe decline in non-verbal cognitive functions. ${ }^{25} \mathrm{It}$ subsequently became clear that the progressive aphasic syndrome consists of two, fairly distinct, clinical subsets. One subset of patients has a progressive non-fluent aphasia (with phonological and syntactic deficits) at onset and shows a late and minimal decline in non-verbal cognitive functions. The topogra- phy of pathology in them is a predominant asymmetric involvement of the left perisylvian region. ${ }^{24}$ They have been designated as progressive non-fluent aphasia (PA). ${ }^{41}$ Another subset of patients present with a progressive fluent aphasia and profound anomia, secondary to a breakdown in semantic memory. They progress more rapidly than PA to involve the non-verbal cognitive functions. The topography of lesion in such cases predominantly involves anterolateral portions of temporal lobe. This group of patients have been labelled semantic dementia (SD). ${ }^{23}$ Less common focal presentations, such as progressive prosopagnosia, ${ }^{43}$ are also known to occur when focal atrophy predominantly involves the right temporal lobe. Although the progressive aphasic syndromes (PA and SD) and the frontotemporal degeneration syndrome differ clinically, they share common features in pathology. These include focal lobar atrophy and a histology showing neuronal loss from superficial cortical layers with spongiosis and variable degrees of gliosis with or without Pick cells or Pick bodies. ${ }^{42} 44$

The recognition that patients with similar histological changes, could present with either a progressive aphasic or a progressive frontotemporal behavioural syndrome, and that with time features of both can appear in the same patient, encouraged the origin of the broader term of frontotemporal dementia (FTD). The clinical criteria for diagnosis of FTD along with criteria to describe the accompanying histological changes, were laid in the consensus statement of the Lund Manchester groups. ${ }^{22}$ and have recently been modified. ${ }^{45}$

The realisation that it is the topographical distribution of atrophy that determines the clinical syndrome, encouraged further differentiation. Snowden et $a l^{41}$ suggested further modifications by introducing the term frontotemporal lobar degeneration (FTLD) to specify an anatomically circumscribed primary degeneration which may be accompanied by any one of the three possible histological patterns. ${ }^{22}{ }^{45}$ They suggested that the use of the term frontotemporal dementia be restricted to describe a clinical syndrome. Thus, according to this description, frontotemporal dementia is the commonest clinical phenotype of FTLD, others being progressive non-fluent aphasia and semantic dementia. Others have favoured the use of the terms frontal variant FTD and temporal variant FTD, the second being synonymous with the progressive aphasic syndrome of SD. ${ }^{21}$ Kertesz, on the other hand, has argued that the term frontotemporal dementia (or degeneration), has restricted clinical and topographical conotations. Emphasising the commonality of the underlying histology, his group proposed to unify all the different clinical and pathological phenotypes of non-Alzheimer dementias under the term "Pick complex". ${ }^{46}$

The evolution of the term CBD is less complex. It was first described as a clinicopathological entity by Rebeiz et $a l,{ }^{47}$ who called it corticodentatonigral degeneration with neuronal achromasia. As further clinical ${ }^{48}$ and 
pathological $^{8} 9$ findings accumulated, Riley et $a l^{48}$ coined the term cortical-basal ganglionic degeneration (CBD). Thus according to the current viewpoint, as discussed at the beginning of this report, CBD is considered a fairly distinct clinical entity with certain typical pathological features.

Although an earlier report ${ }^{47}$ failed to recognise the cognitive decline or language disorder, recent reports have noted dementia ${ }^{789}$ and progressive aphasia ${ }^{8-1244}$ in patients with CBD. Indeed, two patients of Bergeron et $a l{ }^{7}$ one patient of Kertesz and Munoz, ${ }^{49}$ and one patient of Lippa et $a l,{ }^{8}$ who showed histological features of CBD on necropsy, presented with dementia. The dementia in all cases has been of a frontal or frontotemporal pattern. Thus, FTD is the principal clinical differential diagnosis for such cases. In the absence of typical motor features, there seem to be no definite clinical characteristics in the dementia to help make the distinction. On a similar note, one patient of Bergeron et $a l,^{7}$ three patients of Kertesz and Munoz, ${ }^{49}$ and the patients reported by Lippa et al, ${ }^{8}$ Sakurai et al,$^{10}$ Ikeda et $a l,{ }^{11}$ and Yoshimura et $a l^{12}$ who presented with progressive aphasia, showed histological features of CBD on necropsy. Thus CBD should also be considered in the differential diagnosis for progressive aphasia. ${ }^{44}$ Whereas the nature of aphasia in most cases had been non-fluent, the patient reported by Yoshimura et $a l^{12}$ had fluent aphasia. Nearly all cases of CBD presenting with progressive aphasia (fluent or non-fluent), eventually evolved to show impairment of other cognitive spheres. $^{7-12}$ So far, most reported cases of CBD, with either of the atypical cognitive presentations discussed above, have gone on to develop obvious asymmetric extrapyramidal and motor features that are typical for CBD. ${ }^{4-1250}$ Surprisingly, despite the fact that

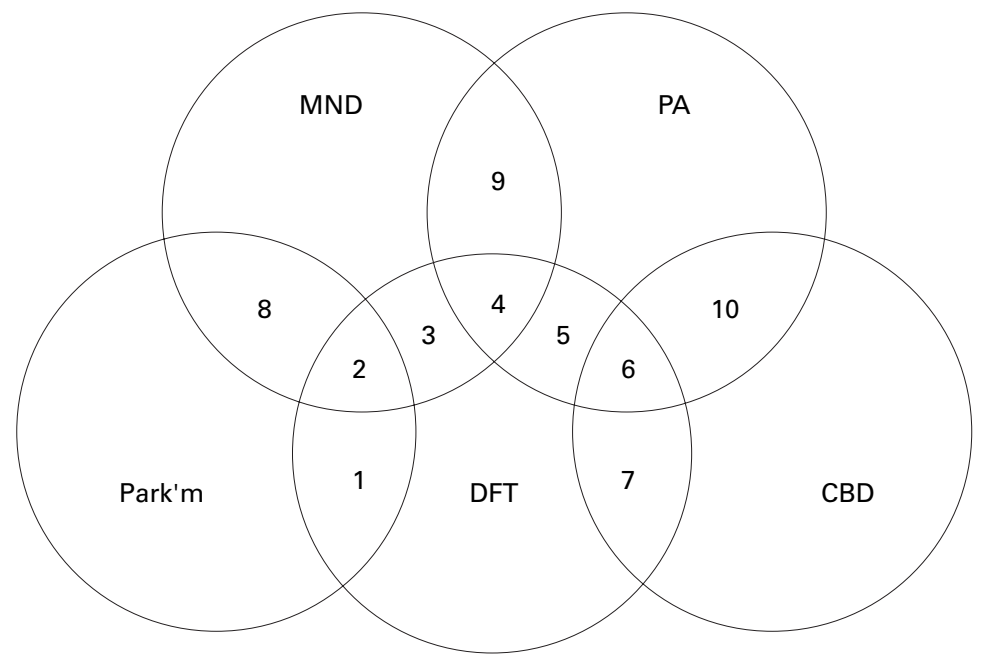

Figure 6 Degenerative syndromes and their overlaps with FTD resulting in various, clinical syndromes of non-Alzheimer dementias. Major degenerative syndromes: Park' $m=$ Parkinsonism; $M N D=$ motor neuron disease; $P A=$ progressive aphasia $; C D=$ corticobasal degeneration; $D F T=$ dementia of frontal type. Overlap non-Alzheimer dementia syndromes: $1=$ frontotemporal dementia and parkinsonism (FTDP);2=syndrome of parkinsonism, amyotrophy, and dementia complex; $3=M N D$ with frontal dementia ${ }^{52} ; 4=M N D$ with amyotrophy, and dementia complex; $3=M N D$ with frontal dementia $a^{52} ; 4=M N D$ with
dementia and aphasia ${ }^{53} ; 5=$ progressive aphasia with frontal dementia (non-fluent PA or semantic dementia); $6+7=C B D$ with prominent dementia; $8=$ parkinsonism with amyotrophy $y^{54} ; 9=M N D$ with aphasia ${ }^{53} ; 10=C B D$ with aphasia. Reports suggest that syndromes 9 and 10 progress to syndromes 4 and 6 respectively. Follow up studies of syndrome 8 are not available. pathologically both our cases did show significant basal ganglionic involvement, even after 7 years (for case 1) and 4 years (for case 2) of illness, neither of them showed the typical clinical features of CBD, and would perhaps still carry a premortem diagnosis of FTD. Similar findings have been reported by Kertesz and Munoz. ${ }^{49}$

Although the number of FTD-CBD overlap cases remain fairly small, some provisional conclusions can be drawn. Firstly, histological features thought to be typical of $\mathrm{CBD}$ can remain unaccompanied by the typical clinical features of CBD during the patient's lifetime as in the cases that are the subject of this report and that of Kertesz and Munoz. ${ }^{49}$ Secondly, the histological features thought to be typical of CBD can clearly accompany the clinical syndrome of FTD, as illustrated by the cases in this and other ${ }^{69}$ reports. Finally, patients whose illness begins with clinical features suggestive of FTD or progressive aphasia can evolve to manifest clinical features characteristic of CBD as noted by Kertesz and Munoz in 14 of their patients ${ }^{49}$ and by others. ${ }^{10-12}$ These findings suggest that the currently accepted clear distinction between $\mathrm{CBD}$ and FTD is incorrect.

CLASSIFYING NON-ALZHEIMER DEMENTIAS: PHENOTYPE VERSUS GENOTYPE?

As discussed above and emphasised by others ${ }^{51}$ the clinical presentation in non-Alzheimer dementias, is determined by the topographical distribution of the pathology, rather than the type of histology. The major clinical syndromes and their variants ${ }^{52-54}$ produced by overlap between them, are illustrated in fig 6. By contrast with these distinct topographically determined clinical phenotypes, the histological phenotypes found in the patients with nonAlzheimer dementias seem to be less distinctive. The histological findings which include Pick cells, Pick bodies, achromatic neurons, tau positive gial and/or neuronal inclusions, basophilic inclusions in basal ganglia, ubiquitinated tau negative non-eosinophilic neuronal inclusions, non-specific neuronal loss, spongiosis, plaques, and tangles, occur in various combinations and are shared to varying extents by the different clinical syndromes. This leads to complex interactions between the clinical syndromes and the histology. Although some clinical syndromes or some histological features may be more characteristic for a particular disorder, none are probably exclusive. The reason for lack of exclusive histological responses is not known. It could be either due to a limited histological repertoire of brain to various different aetiological insults or a result of a common aetiopathogenetic mechanism shared by various non-Alzheimer dementias.

The current clinicopathological approach for the classification of the non-Alzheimer dementias is unlikely to prove to be the best way to classify these diseases in the long term. Recently, Pasquier and Delacourte have advanced a molecular classification for nonAlzheimer dementias. ${ }^{55}$ Although useful, it is primarily based on the histology at necropsy. 
For the purpose of management, prognosis and selection of patients for clinical trials, it is essential to have a classification for nonAlzheimer dementias that can be applied in vivo.

Although the frequency of familial occurrence of FTD is thought to be low, reliable epidemological evidence shows a family history in up to $40 \%$ of cases. ${ }^{56}$ Whereas most cases of CBD reported so far have been sporadic without any family histories, patient 2, reported here, had a history of dementia in the family. Although this could not be investigated any further, this finding becomes interesting in the light of recent reports by Brown et $a P^{75}$ who found a relatively early onset dementing disorder in 15 members of two families. At least one member from each family, on necropsy, had pathology consistent with CBD. ${ }^{57}$ They also noted that within a single pedigree, members presented with clinical syndromes of frontal lobe dementia or CBD. ${ }^{58}$ This may reflect variations in topographical distribution of the pathological process. Thus it seems likely that some cases with clinical features of CBD (especially the ones that overlap with FTD) may have a genetic basis. Advances in the molecular genetics of non-Alzheimer dementias are likely to ultimately offer a better basis for classification of these disorders. At least two clinical phenotypes of FTD, one with prominent parkinsonism (FTDP) ${ }^{56}$ 59-62 $^{\text {and the other }}$ with progressive aphasia, ${ }^{63}$ have already been localised to a defect on chromosome 17q21-22 in some families and further, the former phenotype has also been linked to a tau protein gene mutation. It is possible that the many clinical variants of FTD may reflect different phenotypic expression of a single genotype. Alternatively, more genotypes may be discovered in future to explain the variations in clinical phenotypes. Until such time that the molecular genetics and the aetiopathogenetic mechanisms are better understood, we think that it may be better to distinguish between clinical and pathological levels of description and to classify cases of non-Alzheimer dementias, in vivo, on the basis of the clinical phenotype.

1 Lange AE, Riley DE, Bergeron C. Cortical-basal ganglionic degeneration. In: Calne DB, ed. Neurodegenerative diseases. Philedelphia: WB Saunders, 1994:887-94.

2 Ikeda K. Basic pathology of corticobasal degeneration. Neu ropathology 1997;17:127-33.

3 Rinne JO, Lee MS, Thompson PD, et al. Corticobasal degeneration. A clinical study of 36 cases. Brain 1994;117: d183-96.

4 Schneider JA, Watts RL, Gearing M, et al. Corticobasal degeneration: neuropathologic and clinical heterogeneity. degeneration: neuropathologi

5 Wakabayashi K, Takahashi H. Similarities and differences among progressive supranuclear palsy, corticobasal degeneration and Pick disease. Neuropathology 1996;16:262-8.

6 Clark AW, Manz HJ, White CL, et al. Cortical degeneration with swollen chromatolytic neurons: its relationship to Pick's disease. F Neuropathol Exp Neurol 1986;45:268-84

7 Bergeron C, Pollanen MS, Weyer L, et al. Unusual clinical presentations of cortical-basal ganglionic degeneration. Ann Neurol 1996;40:893-900.

8 Lippa CF, Smith TW, Fontneau N. Corticonigral degeneration with neuronal achromasia. A clinicopathologic study of two cases. I Neurol Sci 1990;98:301-10.

9 Lippa CF, Cohen R, Smith TW, et al. Primary progressive aphasia with focal neuronal achromasia. Neurology 1991; 41:882-6.

10 Sakurai $\mathrm{Y}$, Hashida $\mathrm{H}$, Uesugi $\mathrm{H}$, et al. A clinical profile of corticobasal degeneration presenting as primary progressive aphasia. Eur Neurol 1996;36:134-7.
11 Ikeda K, Akiyama H, Iritani S, et al. Corticobasal degeneration with primary progressive aphasia and accentuated cortion with primary progressive aphasia and accentuated cortical lesion in superior temporal gyrus: case
review. Acta Neuropathol (Berl) 1996;92:534-9.

12 Yoshimura I, Yoshimura N, Hayashi S, et al. A clinicopathological study of senile dementia presenting as progressive sensory aphasia: a variant of corticobasal degeneration. $\mathscr{f p n}$ f Clin Psychiatry 1995;24:193-200. (In Japanese.)

13 Tsuchiya K, Ikeda K, Uchihara T, et al. Distribution of cerebral cortical lesions in corticobasal degeneration: a clinicopathological study of five autopsy cases in Japan. Acta Neuropathol (Berl) 1997;94:416-24.

14 Yamauchi H, Fukuyama H, Nagahama Y, et al. Atrophy of the corpus callosum, cortical hypometabolism, and cognitive impairment in corticobasal degeneration. Arch Neurol 1998;55:609-14.

15 Cambier J, Masson M, Dairon R, et al. Etude anatomoclinique d' une forme parietale de maladie de Pick. Rev Neurol (Paris) 1981;137:33-8.

16 Lange AE, Bergeron C, Pollanen MS, et al. Parietal Pick disease mimicking cortical-basal ganglionic degeneration. Neurology 1994;44:1436-40.

17 Greene JDW, Patterson K, Xuereb J, et al. Alzheimer disease and nonfluent progressive aphasia. Arch Neurol 1996;53: 1072-8.

18 Hodges JR, Patterson K, Oxbury S, et al. Semantic dementia: progressive fluent aphasia with temporal lobe atrophy. Brain 1992;115:1783-806.

19 Rosser A, Hodges JR. Initial letter and semantic category fluency in Alzheimer's disease, Huntington's disease and

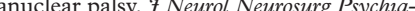
try 1994;57:1389-94.

20 Hodges JR, Paterson K. Nonfluent progressive aphasia and semantic dementia: a comparative neuropsychological study. Fournal of the International Neurophychological Society 1996;2:511-24.

21 Hodges JR, Patterson K, Ward R, et al. The differentiation of semantic dementia and frontal lobe dementia (temporal and frontal variants of frontotemporal dementias) from early Alzheimer's disease: a comparative neuropsychological study. Neuropsychology 1993;13:31-40.

22 The Lund and Manchester Groups. Clinical and neuropathological criteria for frontotemporal dementia. $7 \mathrm{Neu}$ rol Neurosurg Psychiatry 1994;57:416-8.

23 Snowden JS, Griffiths H, Neary D. Semantic dementia: a form of circumscribed atrophy. Behav Neurol 1989;2:16782.

24 Mesulam MM. Slowly progressive aphasia without generalised dementia. Ann Neurol 1982;11:592-8.

25 Weintraub S, Rubin NP, Mesulam MM. Primary progressive aphasia: longitudinal course neuropsychological profile and language features. Arch Neurol 1990;47:1329-35.

26 Neary D, Snowden JS, Northen B, et al. Dementia of frontal Neary D, Snowden JS, Northen B, et al. Dementia of frontal
lobe type. $\mathcal{F}$ Neurol Neurosurgery Psychiatry 1986;49:16374

27 Papp MI, Kahn JE, Lantos PL. Glial cytoplasmic inclusions in the CNS of patients with multiple system atrophy (striatonigral degeneration ollivopontocerebellar atrophy and Shy-Drager syndrome). F Neurol Sci 1989;94:79-100.

28 Wakabayashi K, Oyanagi K, Makifuchi T, et al. Corticobasal degeneration: etiopathological significance of the cytoskeletal alterations. Acta Neuropathol 1994;87:545-53.

29 Mori H, Nishimura M, Namba Y, et al. Corticobasal degeneration: a disease with widespread appearance of abnormal tau and neurofibrillary tangles, and its relation to progressive supranuclear palsy. Acta Neuropathol 1994;88: 113-21.

30 Hauw JJ, Daniel SE, Dickson DS, et al. Preliminary NINDS neuropathologic criteria for Steele-Richarson-Olzewski syndrome (progressive supranuclear palsy). Neurology 1994:44:2015-19.

31 Esiri MM, Hyman BT, Beyreuther K, et al. Ageing and dementia. In: Graham DI, Lantos PL, eds. Greenfield's neuropathology. London: Arnold, 1997;153-233.

32 Hori A, Volles E, Witzke R, et al. Pick's disease of early onset with neurologic symptomatology rapid course and nigrosriatal degeneration. Clin Neuropathol 1983;2:8-15.

33 Hulette CM, Crain B. Lobar atrophy without Pick bodies. Clin Neuropathol 1992;11:151-6.

34 Berrios GE, Girling DM. Introduction: Pick disease and the frontal lobe dementias. History of Psychiatry 1994;5:53947.

35 Alzheimer A. Uber eigenartige Krankheitsfalle des sparteren Alters. Zentralblatt fur die Gesamte neurologie und psychiatrie 1910-11:4:356-85.

36 Hodges JR. Pick's disease. In: Burns A, Levy R, eds. Dementia. London: Chapman and Hall, 1993:739-52.

37 Neary D, Snowden JS, Bowen DM, et al. Neuropsychological syndromes in presenile dementia due to cerebra atrophy. $\mathcal{F}$ Neurol Neurosurg Psychiatry 1986;49:163-74.

38 Gustafson L. Frontal lobe degeneration of non-Alzheimer type II- Clinical picture and differential diagnosis. Archives of Gerentology and Geriatrics 1987;6:209-33.

39 Miller BL, Cummings JL, Villanneava-Meyer J, et al. Frontal lobe degeneration- clinical neuropsychological and SPECT characteristics. Neurology 1991;41:1374-82.

40 Knopman DS, Mastri AR, Frey WH, et al. Dementia lacking distinctive histological features: a common non-Alzheimer degenerative disease. Neurology 1990;40:251-6.

41 Snowden JS, Neary D, Mann DMA. Frontotemporal lobar degeneration. London: Churchill Livingstone, 1996.

42 Hodges JR, Garrard P, Patterson K. Semantic dementia. In: Kertesz A, Munoz DG, eds. Pick's disease and Pick complex. New York:Wiley-Liss, 1998:83-104. 
43 Evans JJ, Heggs AJ, Antoun N, et al. Progressive prosopagnosia associated with selective right temporal lobe atrophy. nosia associated with

44 Kertesz A, Hudson L, Mackenzie IR, et al. The pathology and nosology of primary progressive aphasia. Neurology 1994;44:2065-72.

45 Neary D, Snowden JS, Gustafson L, et al. Frontotemporal lobar degeneration. A consensus on clinical diagnostic criteria. Neurology 1998;51:1546-54.

46 Kertesz A. Frontotemporal dementia, Pick disease, and corticobasal degeneration. One entity or 3? 1. Arch Neurol 1997;54:1427-9.

47 Rebeiz JJ, Kolodny EH, Richardson EP Jr. Corticodentatonigral degeneration with neuronal achromasia. Arch Neurol 1968;18:20-33.

48 Riley DE, Lang AE, Lewis A, et al. Cortical-basal ganglionic degeneration. Neurology 1990;40:1203-12.

49 Kertesz A, Munoz DG. Clinical and pathological overlap in Pick complex. In: Kertesz A, Munoz DG, eds. Pick's disease Pick complex. In: Kertesz A, Munoz DG, eds. Pick's disea

50 Bergeron C, Davis A, Lang AE. Corticobasal ganglionic degeneration and progressive supranuclear palsy presentdegeneration and progressive supranuclear palsy present-

51 Neary D. Frontotemporal degeneration, Pick disease, and corticobasal degeneration. One entity or 3? 3. Arch Neurol 1997;54:1425-7.

52 Kew J, Leigh N. Dementia with motor neurone disease. Baillieres Clin Neurol 1992;1:611-26.

53 Rakowicz WP, Hodges JR. Dementia and aphasia in motor neuron disease: an underrecognised association? $\mathcal{F}$ Neurol Neurosurg Psychiatry 1998;65:881-9.

54 Qureshi AI, Wilmot G, Dihenia B, et al. Motor neuron disease with parkinsonism. Arch Neurol 1996;53:987-91.
55 Pasquier F, Delacourte A. Non-Alzheimer degenerative dementias [in process citation]. Curr Opin Neurol 1998;11:

56 Stevens M, van Duijn CM, Kamphorst W, et al. Familial aggregation in frontotemporal dementia. Neurology 1998; 50:1541-5.

57 Brown J, Lantos PL, Roques P, et al. Familial dementia with swollen achromatic neurons and corticobasal inclusion bodies: a clinical and pathological study. F Neurol Sci 1996; 135:21-30.

58 Brown J, Lantos PL, Rossor MN. Familial dementia lacking specific pathological features presenting with clinical features of corticobasal degeneration. 7 Neurol Neurosurg Psychiatry 1998;65:600-3.

59 Wijker M, Wszolek ZK, Wolters EC, et al Localization of the gene for rapidly progressive autosomal dominant parkinsonism and dementia with pallido-ponto-nigral degeneration to chromosome 17q21. Hum Mol Genet 1996;5:151-4

60 Spillantini MG, Murrell JR, Goedert M, et al. Mutation in the tau gene in familial multiple system tauopathy with the tau gene in familial multiple system tauopathy with presen.

61 Hutton M, Lendon CL, Rizzu P, et al. Association of missense and 5'-splice-site mutations in tau with the inherited dementia FTDP-17. Nature 1998;393:702-5.

62 Dumanchin C, Camuzat A, Campion D, et al. Segregation of a missense mutation in the microtubule-associated protein tau gene with familial frontotemporal dementia and parkinsonism. Hum Mol Genet 1998;7:1825-9.

63 Lendon CL, Lynch T, Norton J, et al. Hereditary dysphasic disinhibition dementia: a frontotemporal dementia linked to 17q21-22. Neurology 1998;50:1546-55. 